\title{
ENSAYO EXPLORATORIO DE MÉTODOS E INTERACCIONES DE ELEMENTOS EN LA APLICACIÓN DE FERTILIZANTES EN EL CULTIVO DE MAIZ1
}

\author{
O. A. Caceros, P. González, I. Hidalgo, B. Moscoso, W.R. Raun ${ }^{2}$
}

\section{COMPENDIO3}

Durante varios años se ha trabajado en la costa Sur con ensayos de fertilización, sin contar a la fecha con respuestas satisfactorias. Con base a lo anterior, se procedieron a realizar ensayos exploratorios para la evaluación de métodos de aplicación de fertilizantes y las Interacciones de los distintos elementos con los siguientes objetivos: Establecer si existe respuesta a la aplicación de fertilizantes, establecer el mejor método de aplicar N, P, S y estiércol, y evaluar la urea versus sulfato de amonio y observar efectos en rendimiento del maíz a la interacción del fósforo con diferentes elementos. Se observó un efecto positivo con la aplicación de fertilizantes, aplicados juntos en banda, principalmente sulfato de amonio y triple superfosfato, efecto que en otros ensayos ha sido debido aun mayor sistema radicular dentro de la banda donde los elementos se encuentran juntos. Además, debido a el cambio del $\mathrm{pH}$ en la rizosphera, se puede aumentarla disponibilidad del $\mathrm{H}_{2} \mathrm{PO}_{4}$. Con respecto alas fuentes de nitrógeno, sulfato de amonio, presentó una mayor respuesta a la fertilización en comparación con aplicaciones de urea, pero el uso de sulfato de amonio aumentaría la acidez M suelo. Por esta razón, aplicaciones del $\mathrm{CaSO}_{4}$ (sal neutra) fueron estudiadas como fuente de azufre y como una alternativa que aplicada junto con el fósforo permitiría una mayor disponibilidad del $\mathrm{H}_{2} \mathrm{PO}_{4}$, por los productos reactivos Iniciales que se forman en el micro ambiente de la banda. La respuesta a la fertilización de P, S y N observados en estos ensayos indican que existen interacciones antagonísticas entre estos elementos. Estudios futuros van a requerir una evaluación más profunda del método usado (bandas juntas o aparte) y las fuentes a emplearse.

Palabras clave adicionales: Zea mays L., azufre, fósforo, nitrógeno, ácido acético, potasio, estiércol, fertilización.

\section{INTRODUCCIÓN}

Durante varios años en la Subregión IV-3, de Guatemala se han realizado trabajos de fertilización en maíz con NP-K, sin encontrar a la fecha respuestas satisfactorias. En algunos casos, como el parcelamiento La Máquina, no se ha determinado una respuesta económica a la fertilización, es decir, que el incremento mínimo en rendimiento, no cubre económicamente la inversión hecha en esta práctica; por lo que actualmente no se recomienda fertilizar.

En base a lo anterior, al equipo de Prueba de Tecnología, conjuntamente con técnicos del Programa de Maíz, enfocó la investigación explorando métodos e interacciones de elementos en la aplicación de fertilizantes

\begin{abstract}
Soil fertility studies have been conducted on the southern coastal plain of Guatemala for several years without showing significant response to phosphorus and sulfur fertilizer applications. In 1987, various exploratory trials were established to evaluate various methods of application for phosphorus, sulfur and nitrogen. Nitrogen sources evaluated were ammonium sulfate and urea. Beef manure was also evaluated in combination with ammonium sulfate. Various other N (as urea), P, S combinations were Included in this trial using $\mathrm{CaSO}_{4}$ as the $\mathrm{S}$ source. Banding phosphorus and nitrogen together especially ammonium sulfate with triple superphosphate provided excellent response across locations. Due to the $\mathrm{pH}$ reduction within the NP band, $\mathrm{H}_{2} \mathrm{PO}_{4}$ availability at lower $\mathrm{pH}$ is Increased as has been demonstrated by others. Ammonium sulfate was by far a better $\mathrm{N}$ source compared to urea when applied alone and as such an $\mathrm{S}$ response was considered probable in 2 or the five locations. Gypsum applications were evaluated both as a source of sulfur and as an alternative to improving $\mathrm{P}$ availability by applying this source with triple superphosphate in a joint band. It was expected that the reaction products from applying gypsum and $\mathrm{P}$ together increase the $\mathrm{P}$ availability since the precipitated products (hypothesized, dicalcium phosphate dihydrate and dicalcium phosphate) would not be fixed and as such slowly available at low $\mathrm{pH}$. Also If by combining triple superphosphate and gypsum in a joint hand, precipitation products DCIP and DCPD would reduce the amount of fertilizer $\mathrm{P}$ complexed with $\mathrm{Fe}$ and $\mathrm{Al}$ hydroxides and or allophane. Antagonistic Interactions were found between $\mathrm{S}$ and $\mathrm{P}$ which suggests that fertilizer $\mathrm{P}$ applications could induce $\mathrm{S}$ deficiencies (where $\mathrm{S}$ was not applied) since $\mathrm{P}$ may be replacing $\mathrm{SO}_{4}$ on the exchange complex of soils thought to have high anion exchange capacities and/or significant amounts of adsorbed $\mathrm{SO}_{4}$.
\end{abstract}

para tratar de encontrar una respuesta económica a esta práctica que beneficie a los agricultores de esta región.

En 1976, en el parcelamiento La Máquina se evaluaron cuatro niveles de Nitr6geno 0, 30, 60 y $90 \mathrm{~kg}$ de $\mathrm{N} / \mathrm{ha}$ y siete formas de aplicación, llegándose a concluir

${ }^{1}$ Presentado en la XXXV Reunión Anual del PCCMCA, San Pedro Sula, Honduras, 1989. Contribución de el Instituto de Ciencia y Tecnología Agrícolas OCTA), Programa de Maíz. Se agradece el apoyo técnico y económico del Centro Internacional de Mejoramiento de Maíz y Trigo (CIMMYT) en la conducción de esta investigación.

2 Delegado Subregional, Técnicos de Prueba de Tecnología, Técnico Programa de Maíz, ICTA; Agrónomo Regional, CIMMYT. Apdo. Postal 231-A, Guatemala, Guatemala, C.A.

${ }^{3} \mathrm{El}$ abstract es traducción del compendio. Elaborado por el Comité Editorial para mostrar el formato de presentaciónde los artículos.

Publicado en Agronomía Mesoamericana, Vol. 1 (1990). 
que no existió respuesta a los niveles de nitrógeno aplicados. Este mismo año también se evaluaron cuatro niveles de nitrógeno en cuatro formas de distribución de plantas, en donde se observó que la fertilización nitrogenada no presenta respuesta consistente, lo que está de acuerdo con otros resultados (Instituto de Ciencia y Tecnología Agrícolas, 1976).

En 1977, se evaluó la "Respuesta a la aplicación de seis niveles de Nitrógeno y Fósforo, usando tres fuentes de nitrógeno en el cultivo de Maíz", en donde se concluyó que no existe diferencia significativa en los sectores A y con respecto a la aplicación de niveles de fertilizante y diferentes fuentes de nitrógeno. En el sector "B" del parcelamiento La Máquina, hubo diferencia estadística entre niveles de fertilización, pero no entre fuentes, considerándose los mejores niveles 100-60 y 150-60 de $\mathrm{N}$ y P 2 0, Estos niveles de fertilización no se consideran económicamente rentables por lo que se continuó el problema de la no respuesta a la fertilización en el parcelamiento La Máquina. Este mismo año, se realizó un estudio sobre la respuesta a la fertilización nitrogenada, mediante la ruptura del suelo a $90 \mathrm{~cm}$ de profundidad y se concluyó que la capa dura o pie de arado como factor individual, no es responsable de la no respuesta económica a la aplicación de fertilizante nitrogenado (Instituto de Ciencia y Tecnología Agrícolas, 1977).

La diferencia en rendimiento entre el testigo y los tratamientos que incluyeron fertilización nitrogenada, nunca fue significativa bajo el punto de vista económico. Se continuó con la investigación y en siembras de segunda se evaluó la "Respuesta a la aplicación de seis niveles de N y P en maíz”, en donde se observó que ninguno de los diferentes niveles de nitrógeno y fósforo incrementaron la producción en las áreas bajo estudio (Instituto de Ciencia y Tecnología Agrícolas, 1977). En 1978, se realizó una "Evaluación de respuesta a la fertilización con N-P-K-S”, en donde se determinó que no se manifiestan efectos positivos marcados entre las interacciones N-P-,K-S. Con la aplicación de urea se alcanzo un incremento de $0.63 \mathrm{tm} / \mathrm{ha}$, en tanto el fósforo presentó un efecto negativo en todas las localidades, que provocó disminución en los rendimientos y con azufre se alcanzaron los mayores incrementos que fueron de 0.67 y $0.65 \mathrm{tm} / \mathrm{ha}$ (Instituto de Ciencia y Tecnología Agrícolas, 1978).

En 1983, se inició la investigación en los parcelamientos Caballo Blanco, Santa Fe y El Rosario, evaluando la respuesta de dos tipos de labranza a la fertilización Nitrofosfórica y densidad de siembra, concluyéndose que la adición de " $\mathrm{N}$ " favorecieron a todos los factores estudiados en cuanto a su respuesta en rendimiento. Los incrementos en rendimiento logrados por cada uno de los factores, no fueron significativos económicamente, siendo la mejor alternativa la mínima labranza, sin aplicación de fertilizante (Instituto de
Ciencia y Tecnología Agrícolas, 1983).

En 1984, se realizó una evaluación agroeconómica de los sistemas Maíz-ajonjolí, Maíz-frijol, de acuerdo a la preparación de suelo y fertilización, encontrándose que para una localidad, el mejor tratamiento fue de 45 $\mathrm{kg}$ de $\mathrm{N} / \mathrm{ha}$, en mínima labranza y con el sistema Maíz-ajonjolí (Instituto de Ciencia y Tecnología Agrícolas, 1984). En 1985, se continuó con este tipo de evaluaciones, en donde para la localidad ubicada en Caballo Blanco, se encontró que tanto para capital limitado como para capital limitado, el mejor tratamiento fue 90 $\mathrm{kg}$ de $\mathrm{N} /$ ha y $45 \mathrm{~kg} \mathrm{P}_{2} \mathrm{O}_{5} / \mathrm{ha}$, en labranza tradicional. Para la localidad 2, ubicada en el mismo parcelamiento, los mejores rendimientos tanto para capital limitado como ¡limitado, se obtuvieron con $90 \mathrm{~kg}$ de N/ha en labranza tradicional, o bien $135 \mathrm{~kg}$ de N/ha en mínima labranza (Instituto de Ciencia y Tecnología Agrícolas, 1985). Ya en 1986, se recomienda evaluar en ensayos agroeconómicos los niveles $90-45 \mathrm{~kg}$ de $\mathrm{N}$ y $\mathrm{P}_{2} \mathrm{O}_{5} / \mathrm{ha}$ con la labranza tradicional, lo que equivale a 3,5 qq de 20-20-0 más 1,5 qq de urea/mz. Para la práctica de cero labranza, se recomienda evaluar $45-45 \mathrm{~kg}$ de $\mathrm{N}$ y $\mathrm{P}_{2} \mathrm{O}_{5}$ ha utilizando $3,5 \mathrm{qq} / \mathrm{mz}$ de 20-20-0 (Instituto de Ciencia y Tecnología Agrícolas, 1986).

Los objetivos consisten en explorar métodos y fuentes de aplicación de fertilizantes en la región IV. También, tratar de encontrar respuestas a preguntas comunes del área, tales como: La no respuesta a la fertilización, efectos del uso de sulfato de amonio en la acidez del suelo y el efecto de método en la aplicación del fósforo con el yeso.

\section{MATERIALES Y MÉTODOS}

\section{Localización del Experimento}

La investigación se realizó en los parcelamientos Caballo Blanco y La Máquina. El parcelamiento Caballo Blanco se encuentra ubicado en el departamento de Retalhuleu a $14^{\circ} 28^{\prime}$ latitud norte y $91^{\circ} 48^{\prime}$ longitud oeste (Guatemala, Instituto Geográfico Nacional, 1972). Según el trabajo realizado por De la Cruz (1982), se encuentra ubicado en la zona de vida "Bosque húmedo Subtropical Cálido", que tiene como características climáticas una precipitación que va de 1.200 a $2.000 \mathrm{~mm}$ anuales y una biotemperatura de $27^{\circ} \mathrm{C}$. La altitud sobre el nivel del mar es de $100 \mathrm{~m}$ (Instituto de Ciencia y Tecnología Agrícolas, 1984).

De acuerdo a la clasificación de reconocimiento de suelos (Simmons et al, 1959), los suelos corresponden al grupo del Litoral del Pacífico y a la serie de suelos Ixtán arcilloso, cuyas características son: Suelos profundos, moderadamente bien drenados, desarrollados sobre material de grano fino en un clima cálido, húmedo seco, textura arcillosa color café muy oscura. El suelo es muy 
plástico y pegajoso cuando está húmedo y duro cuando está seco.

El parcelamiento La Máquina se encuentra ubicado en el municipio de Cuyotenango, departamento de Suchitepéquez y San Andrés Villa Seca, departamento de Retalhuleu, aproximadamente a $14^{\circ} 23^{\prime}$ latitud norte y $90^{\circ} 35^{\prime}$ ' 1ongitud oeste, con una altura que varía entre 6 y 150 msnm (Guatemala, Instituto Geográfico Nacional, 1972). Ecológicamente está clasificado como zona tropical seca en la mayor parte de su área, con una temperatura media de $27^{\circ} \mathrm{C}$. La precipitación anual media es de 1,860 mm (1). La serie de suelos predominante (Simmons et al, 1959) es Ixtán, arcilloso y en menor escala Ixtán franco limoso, aunque se encuentran suelos de la serie Champerico en pequeñas áreas asociadas con las anteriores.

\section{Manejo del Experimento}

Se estableció un total de cinco ensayos, tres en el parcelamiento La Máquina y dos en el parcelamiento Caballo Blanco, en terrenos de agricultores colaboradores; quienes prepararon el terreno con un paso de arado y dos de rastra. El diseño estadístico utilizado fue de bloques al azar con 14 tratamientos y 4 repeticiones. La unidad experimental consistió en seis surcos de seis metros de largo, separados a 0,90 m entre sí y $0,50 \mathrm{~m}$ entre plantas, para hacer un área de 32,4 m cuadrados por parcela y un área total del ensayo de $1.184,4 \mathrm{~m}$ cuadrados.

La siembra se realizó en los primeros 15 días del mes de junio, cuando las lluvias estuvieron establecidas. La fertilización se realizó al momento de la siembra con los siguientes tratamientos y dosis.

1. Sulfato de amonio en banda y $\mathrm{P}_{2} \mathrm{O}_{5}$ en banda aparte. 2. Sulfato de amonio en banda y $\mathrm{P}_{2} \mathrm{O}_{5}$ en banda, juntos. 3. Sulfato de amonio, $\mathrm{P}_{2} \mathrm{O}_{5}$ y $\mathrm{CaSO}_{4}$ en banda, juntos.

4. Sulfato de amonio, $\mathrm{P}_{2} \mathrm{O}_{5} \mathrm{CaSO}_{4}$ y estiércol en banda, juntos.

5. Urea, 46-0-0, $\mathrm{P}_{2} \mathrm{O}_{5}$ y $\mathrm{CaSO}_{4}$ en banda juntos.

6. Sulfato de amonio, $\mathrm{P}_{2} \mathrm{O}_{5}$ y estiércol en banda juntos.

7. Sulfato de amonio en banda aparte, $\mathrm{P}_{2} \mathrm{O}_{5} \mathrm{y}$ estiércol en banda juntos.

8. Urea, 46-0-0 en banda aparte, ácido acético y $\mathrm{P}_{2} \mathrm{O}_{5}$ en banda juntos.

9. Sulfato de amonio y $\mathrm{CaSO}_{4}$ en banda juntos, $\mathrm{P}_{2} \mathrm{O}_{5}$ en banda aparte.

10. Sulfato de amonio y KCI en banda juntos.

11. Urea, 46-0-0 en banda solo (testigo)

12. Sulfato diamónico 8-46-0 y estiércol en banda juntos.

13. Sulfato de amonio solo, en banda.

14. Testigo absoluto, sin fertilización.

\section{Dosis utilizadas}

A. Nitrógeno: $100 \mathrm{~kg}$ de $\mathrm{N} / \mathrm{ha}$

a. Urea 46-0-0

b. Sulfato de Amonio 21-0-0

B. Fósforo: $50 \mathrm{~kg} \mathrm{P}_{2} \mathrm{O}_{5} / \mathrm{ha}$, Superfosfato triple (046-0) (Excepto tratamientos 10-14)

C. Sulfato de calcio: $\left(\mathrm{CaSO}_{4}\right) 57 \mathrm{~kg}$ de $\mathrm{CaSO}_{4} / \mathrm{ha}$

D. Estiércol: $1 \mathrm{t} / \mathrm{ha}$

E. Acido Acético: 10 lt/ha

F. Cloruro de Potasio: $(\mathrm{KCl}) 139 \mathrm{~kg}$ de $\mathrm{KC} 1 / \mathrm{ha}$

G. $18-46-0: 181 \mathrm{~kg} / \mathrm{ha}$

El control de malezas se efectuó en forma química, utilizando Atrazina a razón de $2 \mathrm{~kg} /$ ha de Gesaprim 80, en forma pre-emergente al cultivo y a la maleza. El control de plagas se realizó utilizando Methamidophos (Tamarón), Methormyl (Lannate) y Phoxim (Volatón), a razón de 1,5 lt/ha y $9 \mathrm{~kg}$ de granulado/ha. La dobla se realizó a los 90 días, cuando el maíz llegó a su madurez fisiológica.

\section{RESULTADOS Y DISCUSIÓN}

Los análisis de varianza de los sitios experimentales (1, 2 y 3) establecidos en La Máquina, se presentan en los cuadros 1,3 y 5 , y en los cuales se presentan altas diferencias significativas entre tratamientos. Tomando en cuenta estas diferencias significativas, se procedió a realizar una comparación múltiple de medias mediante la Prueba de DUNCAN, las cuales se presentan en los cuadros 2, 4 y 6 .

En el sitio experimental 1 se observa cierta tendencia hacia una mejor respuesta a la fertilización, cuando el sulfato de amonio fue aplicado junto con el triple superfosfato, en banda en comparación cuando se aplicó separadamente en bandas los mismos elementos (Trat. 1 contra 2). Situación similar ocurrió cuando se aplicó

Cuadro l. Análisis de varianza en ensayos exploratorios e interacciones de elementos a la aplicación de fertilizantes en el sitio experimental 1, La Máquina. Subregión IV-3, Retalhuleu 1988.

\begin{tabular}{lrlcllll}
\hline \multicolumn{1}{c}{ FV } & GL & SC & CM & FC & 0,05 0,01 Sig \\
\hline Repeticiones & 3 & 12567916.20 & 4189305.40 & 9.37 & 2.85 & $4.34 * *$ \\
Tratamiento & 13 & 17269825.05 & 1328448.08 & 2.97 & 1.96 & $2.59 * *$ \\
Error & 39 & 17438573.79 & & & & \\
\hline
\end{tabular}

$* *$ Significativo al 0,05 y 0,01 niveles de probabilidad. C.V. $\%=17,0$ 
Cuadro 2. Comparación múltiple de medias, mediante la prueba de DUNCAN para el sitio experimental 1, La Máquina. Subregión IV-3, Retalhuleu, 1988.

\begin{tabular}{|c|c|c|}
\hline & Tratamiento & Rendimiento (kg/ha) \\
\hline 06) & (Sulf. Am. $\mathrm{P}_{2} \mathrm{O}_{5}$ y estiércol) & $4.770,4$ a \\
\hline 04) & (Sulf. Am. $\mathrm{P}_{2} \mathrm{O}_{5}, \mathrm{CaSO}_{4}$ y estiércol) & $4.747,0 \quad \mathrm{a}$ \\
\hline 12) & (18-46-0 y estiércol) & $4.546,0 \quad \mathrm{a}$ \\
\hline 08) & (Urea) $\left(\mathrm{P}_{2} \mathrm{O}_{5}\right.$ y Acido Acético) & $4.318,0$ a b \\
\hline 03) & (Sulf. Am. $\mathrm{P}_{2} \mathrm{O}_{5}$ y $\mathrm{CaSO}_{4}$ ) & $4.169,3$ a b \\
\hline 09) & (Sulf. Am. y $\left.\mathrm{CaSO}_{4}\right)\left(\mathrm{P}_{2} \mathrm{O}_{5}\right)$ & $4.131,7$ a b \\
\hline 02) & (Sulf. Am. $\mathrm{P}_{2} \mathrm{O}_{5}$ ) & $4.037,4$ a b \\
\hline 05) & (Urea, $\mathrm{P}_{2} \mathrm{O}_{5}$ y $\mathrm{CaSO}_{4}$ ) & $3.856,6$ a b \\
\hline 10) & (Sulf. Am. y KCl) & $3.610,0 \quad \mathrm{~b} \mathrm{c}$ \\
\hline 14) & Testigo Absoluto & $3.539,1 \quad 1 \mathrm{~b} \mathrm{e}$ \\
\hline 07) & (Sulf Am.) $\left(\mathrm{P}_{2} \mathrm{O}_{5}\right.$ y estiércol) & $3.507,9 \mathrm{bc}$ \\
\hline 13) & (Sulf. Am.) & $3.492,9$ b c \\
\hline 01 & (Sulf. Am.) $\left(\mathrm{P}_{2} \mathrm{O}_{5}\right)$ & $3.430,3 \mathrm{~b} \mathrm{e}$ \\
\hline 11) & (Urea) & $2.735,9 \mathrm{c}$ \\
\hline
\end{tabular}

( ) - Indica que los elementos se aplicaron en bandas separadas.

Cuadro 3. Análisis de varianza en ensayos exploratorios de métodos e interacciones de elementos en aplicaciones de fertilizantes para el sitio experimental 2 La Máquina. Subregión IV-3, 1988.

\begin{tabular}{lllclllll}
\hline \multicolumn{1}{c}{ FV } & GL & SC & CM & FC & $\mathbf{0 , 0 5}$ & $\mathbf{0 , 0 1}$ & Sig \\
\hline Repeticiones & 3 & 148185,89 & 493950,63 & 5,80 & 2,85 & 4,34 & $* *$ \\
Tratamiento & 13 & 6006479,08 & 462036,85 & 5,43 & 1,96 & 2,59 & $* *$ \\
Error & 39 & 3321324,68 & & & & & \\
\hline
\end{tabular}

C.V. $\%=5,5$

sulfato de amonio, triple superfosfato y estiércol en banda junto, en comparación a la aplicación de sulfato de amonio en banda aparte, triple superfosfato y estiércol en banda junto (Trat. 6 contra 7). Con respecto a las fuentes de nitrógeno, el sulfato de amonio presentó cierta tendencia hacia una mayor respuesta a la fertilización que la urca (Trat. 11 contra 13).

Observando el comportamiento del testigo absoluto, se puede ver que en este caso, superó a los tratamientos sulfato de amonio en banda aparte, triple sulfato y estiércol en banda juntos, sulfato de amonio, sulfato de amonio en banda aparte y triple superfosfato en banda aparte y urea.

En el sitio experimental 2, también se observa que aplicaciones de sulfato de amonio y fósforo en banda juntos, presentan tendencia a una mejor respuesta ala fertilización, en comparación a cuando dichos elemen- tos son aplicados en banda aparte (Trat. 1 contra 2). El tratamiento sulfato de amonio, fósforo y estiércol en banda juntos, también presenta una tendencia a una mejor respuesta a la fertilización que cuando se aplicó sulfato de amonio aparte y fósforo y estiércol juntos (Trat. 6 contra 7)

La aplicación de sulfato de amonio solo, presentó una tendencia a incrementar los rendimientos en comparación con la aplicación de Urea sola (Trat. 11 contra 13); esto corroborado con la respuesta obtenida con el tratamiento sulfato de amonio, fósforo y sulfato de calcio en banda juntos, que mostró una tendencia hacia una mayor respuesta en comparación con Urea, fósforo y sulfato de calcio juntos (Trat 3 contra 5).

En el sitio experimental 3, se obtuvieron algunas respuestas diferentes a las de los sitios anteriores, tal es el caso del tratamiento urea aparte y fósforo más ácido acético en banda junto, que es el que presenta la mejor

Cuadro 4. Comparación múltiple de medias mediante la prueba de DUNCAN para el sitio experimental 2, Subregión IV-3, Retalhuleu 1988.

Tratamiento $\quad$ Rendimiento $(\mathrm{kg} / \mathrm{ha})$

04) (Sulf. Am. $\mathrm{P}_{2} \mathrm{O}_{5}, \mathrm{CaSO}_{4}$ y estiércol) 5.766,6 a

02) (Sulf. Am. - $\mathrm{P}_{2} \mathrm{O}_{5}$ ) $5.576,9 \mathrm{ab}$

06) (Sulf. Am. $\mathrm{P}_{2} \mathrm{O}_{5}$, - estiércol) $5551,9 \mathrm{a} \mathrm{b}$

13) (Sulf. Am.)

03) (Sulf. Am., $\mathrm{P}_{2} \mathrm{O}_{5}$ y $\mathrm{CaSO}_{4}$ ) 5338.1 b c

10) (Sulf. Am. y KCI) 5335.6 b c

07) (Sulf. Am.) ( $\mathrm{P}_{2} \mathrm{O}_{5}$, estiércol) $\quad 5297.5 \mathrm{bc}$

11) (Urea) $\quad 5287.6 \mathrm{bc}$

01) (Sulf Amonio) $\left(\mathrm{P}_{2} \mathrm{O}_{5}\right) \quad 5276.8 \mathrm{~b} \mathrm{c}$

09) (Sulf Am. y $\left.\mathrm{CaSO}_{4}\right)\left(\mathrm{P}_{2} \mathrm{O}_{5}\right) \quad 5252.1 \mathrm{~b} \mathrm{c}$

05) (Urea, $\mathrm{P}_{2} \mathrm{O}_{5} \quad$ y CaSO $_{4}$ ) 52419 b e

08) (Urea) $\left(\mathrm{P}_{2} \mathrm{O}_{5}\right.$, ácido acético) 52293 b c

12) (18-46-0 y estiércol) $5117.4 \mathrm{c}$

14) Testigo Absoluto 4255.9 d

Cuadro 5. Análisis de varianza en ensayos exploratorios de métodos e interacciones de elementos en la aplicación de fertilizante en el sitio experimental 3, La Máquina. Subregión IV-3, Retalhuleu 1988.

\begin{tabular}{lcccccccc}
\hline \multicolumn{1}{c}{ FV } & GL & SC & CM & FC & $\mathbf{0 , 0 5}$ & $\mathbf{0 , 0 1}$ & Sig \\
\hline Repeticiones & 3 & 503176.772 & 167725.591 & 2.96 & 2.85 & 4.34 & $*$ \\
Tratamiento & 13 & 2870179.953 & 220783.073 & 3.90 & 1.96 & 2.59 \\
Error & 39 & 2207969.076 & 566124.592 & & & & \\
\hline
\end{tabular}

$\mathrm{CV}, \%=4,5$ 
Cuadro 6. Comparación múltiple de medias mediante la prueba de DUNCAN para el sitio experimental 3, Subregión IV-3, Retalhuleu 1988

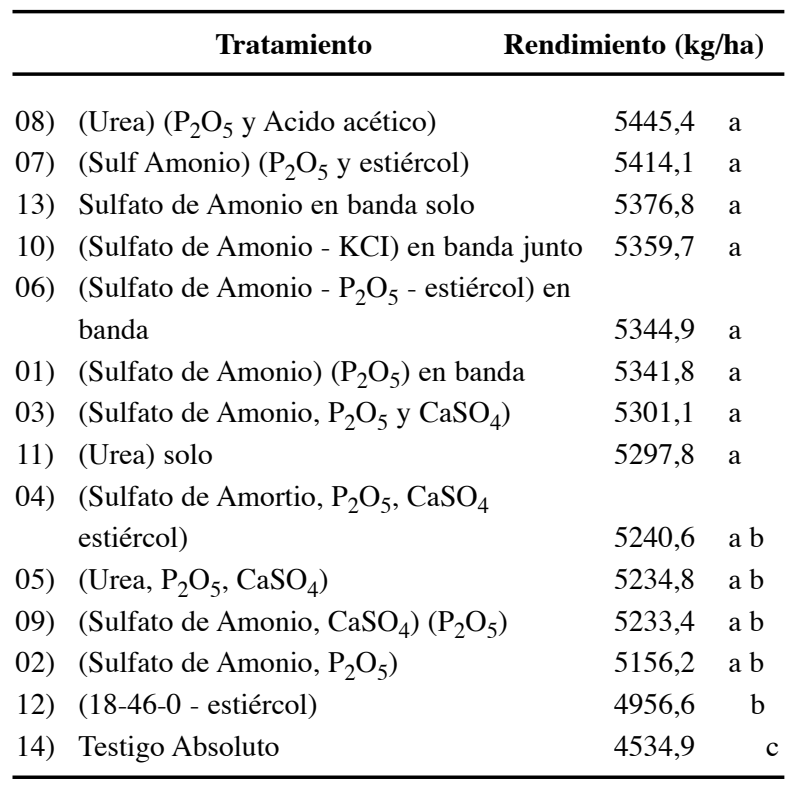

tendencia hacia una mayor respuesta a la fertilización. Esta respuesta está de acuerdo a los conceptos desarrollados por Watanabe, 1966, donde cargas positivas en arcillas alofánicas fueron neutralizadas, usando ácido húmico. Con el uso de ácido acético dentro de la banda de fósforo, se esperaba reducir la fijación de fósforo por el alófano; de este modo, alguna degradación de la estructura del alófano sería obtenida, esto en efecto podría mejorar el uso eficiente del fósforo. Aunque es hipotético, de hecho requerirá futuras evaluaciones.

También se observó, que aplicaciones de sulfato de amonio y fósforo en banda aparte, presentaban tendencias hacia una mejor respuesta a la fertilización (Trat. 1 contra 2). Situación similar se presentó para las aplicaciones de sulfato de amonio aparte y fósforo más estiércol juntos en banda, en comparación con el tratamiento en que se aplicaron estos mismos elementos en banda juntos (Trat. 6 contra 7). Aplicaciones de sulfato de amonio en banda solo, se obtuvo una tendencia a obtener una mejor respuesta, que aplicando solamente urea.

El testigo absoluto fue superado por el resto de los tratamientos evaluados, lo que nos da un índice de respuesta a la fertilización. Los análisis de varianza de estos sitios experimentales (4 y 5), establecidos en el parcelamiento Caballo Blanco, se presentan en los cuadros 7 y 9 , en los cuales se observan altas diferencias significativas entre tratamientos; razón por la cual se procedió a efectuar la comparación múltiple de medias, utilizando la Prueba de DUNCAN, los que se presentan en los Cuadros 8 y 10, respectivamente. En este cuarto si-
Cuadro 7. Análisis de varianza en ensayos expiaratorlos de métodos e interacciones de elementos en aplicaciones de fertilizantes en el sitio experimental 4, Caballo Blanco, Subregión IV-3, Retalhuleu 1988

\begin{tabular}{|c|c|c|c|c|c|}
\hline $\mathbf{F}$ & GL & SC & CM & FC & $\begin{array}{c}\text { Ft } \\
0,05 \quad 0,01 \quad \text { Sig }\end{array}$ \\
\hline \multicolumn{2}{|c|}{ Repeticiones } & 3 & 29504,22 & 9834,74 & $4 \quad 0,12$ \\
\hline 2,85 & 4,34 & NS & & & \\
\hline \multicolumn{2}{|c|}{ Tratamiento } & 13 & 6194536,4 & & 476502,80 \\
\hline 5,6 & 1,96 & 2,59 & $* *$ & & \\
\hline Error & 39 & 33132 & & & \\
\hline
\end{tabular}

$\mathrm{CV}, \%=4,5$

Cuadro 8. Comparación múltiple de medias mediante la prueba de DUNCAN para el sitio experimental 4, Caballo Blanco.

\begin{tabular}{|c|c|c|c|}
\hline & Tratamiento & (kg/ha) & \\
\hline 06) & (Sulf. Am. $\mathrm{P}_{2} \mathrm{O}_{5}$ - estiércol) & 6821,4 & a \\
\hline 10) & (Sulf, Amonio y KG) & 6707,6 & $a b$ \\
\hline 02) & (Sulf, Amonio y $\mathrm{P}_{2} \mathrm{O}_{5}$ & 6620,4 & $\mathrm{abe}$ \\
\hline 05$)$ & (Urea, $\mathrm{P}_{2} \mathrm{O}_{5}$ y $\left.\mathrm{CaSO} 4\right)$ & 6603,1 & $\mathrm{abc}$ \\
\hline 11) & (Urea) & 6569,7 & $\mathrm{abc}$ \\
\hline 08) & (Urea) $\left(\mathrm{P}_{2} \mathrm{O}_{5}\right.$, ácido acético) & 6479,4 & $a b$ e d \\
\hline 03) & (Sulf. Amonio, $\mathrm{P}_{2} \mathrm{O}_{5}$ y CaSO 4 ) & 6464,1 & $\mathrm{bcd}$ \\
\hline 01) & (Sulf. Am.) $\left(\mathrm{P}_{2} \mathrm{O}_{5}\right)$ & 6416,5 & $\mathrm{bcd}$ \\
\hline 09) & (Sulf. Am.) CaSO4) $\left(\mathrm{P}_{2} \mathrm{O}_{5}\right)$ & 6345,0 & $\mathrm{bcc}$ \\
\hline 04) & (Sulf. Am., $\mathrm{P}_{2} \mathrm{O}_{5}, \mathrm{CaSO} 4$ y estiércol) & 6337,5 & $\mathrm{bcd}$ \\
\hline 13) & (Sulf. Am.) & 6283,4 & $\mathrm{~cd}$ \\
\hline 06) & (Sulf. Am.) $\left(\mathrm{P}_{2} \mathrm{O}_{5}\right.$ y estiércol) & 6273,6 & $\mathrm{~cd}$ \\
\hline 12) & (18-46-0 y estiércol) & 6150,0 & d \\
\hline 14) & Testigo Absoluto & 5376,5 & $\mathrm{c}$ \\
\hline
\end{tabular}

Cuadro 9. Análisis de varianza en ensayos exploratorios de métodos e Interacciones de elementos en la aplicación de fertilizantes en el sitio experimental 5, Caballo Blanco, Subregión IV-3, Retalhuleu 1988

\begin{tabular}{lrllllll}
\hline \multicolumn{1}{c}{ FV } & GL & \multicolumn{1}{c}{ SC } & CM & FC & $\mathbf{0 , 0 5}$ & $\mathbf{0 , 0 1}$ & Sig \\
\hline Repeticiones & 3 & 87385.449 & 291795,15 & 2,72 & 2,85 & 4,34 & $*$ \\
Tratamiento & 13 & 4212594.632 & 324045,74 & 3,02 & 1,96 & 2,59 \\
Error & 39 & 4185626.929 & & & & & \\
\hline
\end{tabular}

$\mathrm{CV}, \%=4,9$ 
Cuadro 10. Comparación múltiple de medias mediante la prueba de DUNCAN para el sitio experimental 5, Caballo Blanco, Subregión IV-3, Retalhuleu.

\begin{tabular}{|c|c|c|}
\hline & Tratamiento & Rendimiento (kg/ha) \\
\hline 03) & (Sulf Am. $\mathrm{P}_{2} \mathrm{O}_{5}$ y $\mathrm{CaSO}_{4}$ ) & 6972,3 a \\
\hline 08) & (Urea) $\left(\mathrm{P}_{2} \mathrm{O}_{5}\right.$ y ácido acético) & 6897,2 a b \\
\hline 06) & (Sulf, Amonio, $\mathrm{P}_{2} \mathrm{O}_{5}$ y estiércol) & 6843,9 a b c \\
\hline 01) & $($ Sulf, $\mathrm{Am}),\left(\mathrm{P}_{2} \mathrm{O}_{5}\right)$ & 6839,0 a b c \\
\hline 02) & (Sulf Am, y $\mathrm{P}_{2} \mathrm{O}_{5}$ ) & 6825,6 a b e \\
\hline 07) & (Sulf. Am., $\mathrm{P}_{2} \mathrm{O}_{5}, \mathrm{CaSO}_{4}$ y estiércol) & 6790,6 a b c \\
\hline 04) & (Sulf. Amonio, $\mathrm{P}_{2} \mathrm{O}_{5}$ y $\mathrm{CaSO}_{4}$ ) & 6736,4 a b c \\
\hline 11) & (Urea) & 6732,2 a b c \\
\hline 09) & (Sulf. Am., $\left.\mathrm{CaSO}_{4}\right)\left(\mathrm{P}_{2} \mathrm{O}_{5}\right)$ & 6530,2 a bc d \\
\hline 10) & (Sulf. Am. y KCl) & 6480,0 \\
\hline 05) & (Urea, $\mathrm{P}_{2} \mathrm{O}_{5}$ y $\mathrm{CaSO}_{4}$ ) & 6451,7 \\
\hline 13) & (Sulf. Am.) & 6409,6 \\
\hline 14) & Testigo Absoluto & 6117,4 \\
\hline 12) & (18-46-0 y estiércol) & 6084,8 \\
\hline
\end{tabular}

tio experimental, nuevamente se observa una tendencia hacia una mejor respuesta a la fertilización, cuando el sulfato de amonio fue aplicadojunto en banda con el fósforo en comparación o contraste cuando se aplicó separadamente en bandas los mismos elementos (Trat. 1 contra 2).

Un comportamiento similar se observó en los tratamientos donde se aplicó sulfato de amonio, fósforo y estiércol, junto en banda versus sulfato de amonio en banda aparte, versus fósforo y estiércol en bandajuntos (Trat 6 contra 7). Es importante hacer notar que cuando se aplicó en banda junto al sulfato de amonio, fósforo y estiércol, se observó una tendencia a incrementar los rendimientos que cuando se aplicó sulfato de amonio y fósforo junto en banda, donde se puede inferir algún efecto positivo del estiércol en el incremento de los rendimientos.

Con respecto a las fuentes de nitrógeno, se puede observar que aplicando solamente urea, se nota una tendencia a una mayor respuesta a la fertilización, que cuando se aplicó únicamente sulfato de amonio. Este comportamiento observado en las fuentes de nitrógeno, también se presentó en los .tratamientos donde se aplicó urea, fósforo y sulfato de calcio en banda junto en comparación con aplicaciones de sul fato de amonio, fósforo y sulfato de calcio, aplicados también en banda junto (Trat. 5 contra 3 ).

Todos los tratamientos donde se aplicó fertilizante, superaron significativamente al testigo absoluto, lo que nos indica que existe para este sitio experimental, una respuesta a la fertilización.

En el sitio experimental 5 existen algunas variantes en las respuesta obtenidas, con respecto a las aplicaciones de sulfato de amonio y fósforo, ya sea junto o sepa- rado en banda. En esta oportunidad, aplicaciones de sulfato de amonio en banda aparte y fósforo aparte, mostraron una tendencia a obtener una mayor respuesta a la fertilización en comparación a aplicaciones en banda junto de estos elementos (Trat. 1 contra 2). Los tratamientos de sulfato de amonio, fósforo y estiércol, aplicado en banda junto, mostró una tendencia positiva a la respuesta de la fertilización en comparación con el tratamiento donde el sulfato de amonio se aplicó aparte, el fósforo y estiércol en banda junto (Trat. 6 contra 7).

Con respecto a las fuentes de nitrógeno, aplicaciones de urea mostraron una tendencia a incrementar los rendimientos en comparación con aplicaciones de sulfato de amonio.

En el cuadro 11 se presentan los resultados de los análisis de suelo y en el cual se observa que el sido experimental 3, localizado en La Máquina, presentó el nivel más bajo de fósforo, pero también fue la que obtuvo la mejor respuesta a las aplicaciones de fósforo. En general, todos los sitios experimentales fueron deficientes en fósforo, excepto el sitio 5 localizado en Caballo Blanco.

Cuadro 11. Análisis de suelo de los cinco sitios experimentales, en los ensayos exploratorios de métodos e interacciones de elementos en aplicaciones de fertilizantes. Subregión IV-3, Retalhuleu 1988.

\begin{tabular}{lrrrrr}
\hline \multicolumn{1}{c}{ Sitios } & & ug/ml & ug/ml & \multicolumn{2}{c}{ meq/100 ml } \\
experimentales & $\mathbf{p H}$ & \multicolumn{1}{c}{$\mathbf{P}$} & $\mathbf{K}$ & $\mathbf{C a}$ & $\mathbf{M g}$ \\
\hline 1. La Máquina & 6,3 & 10,83 & 180 & 10,71 & 2,46 \\
2. La Máquina & 6,4 & 5,83 & 105 & 10,35 & 2,46 \\
3. La Máquina & 6,0 & 2,50 & 120 & 8,10 & 1,68 \\
4. Caballo Blanco & 6,0 & 13,33 & 232 & 7,47 & 1,32 \\
5. Caballo Blanco & 5,8 & 24,00 & 245 & 8,10 & 1,68 \\
\hline
\end{tabular}

\section{DISCUSIÓN}

Se puede decir de esta investigación que aplicaciones de sulfato de amonio y triple superfosfato en banda juntos presentaron una respuesta positiva en el rendimiento, en contraste a cuando estas fuentes fueron aplicadas en banda separada. Estopuedeser debido a dos efectos: 1. Bandas juntas han demostrado mayor eficiencia en el uso del $\mathrm{N}$ y el $\mathrm{P}$, porque en la banda la raíz encuentra estos dos elementos. Es bien conocido que el sistema radicular es más voluminoso en el área (o bandas donde se encuentra el P, especialmente cuando el P es limitante en la producción) donde el fósforo fue aplicado localizado (Olson y Dreier, 1956). 2. Hay un efecto sinergístico, en el cual la asimilación del amonio disminuye el $\mathrm{pH}$ alrededor. de la raíz con el cual se aumenta la 
disponibilidad del ion ortofosfato $\mathrm{H} 2 \mathrm{PO} 4$ el cual es asimilado más que las otras especies de ortofosfatos a un pH bajo (Raun et al. 1987). En suelos ácidos se aumenta la acidez en la rizosfera, cuando el amonio es asimilado por la raíz.

En tres de los sitios experimentales evaluados, las aplicaciones de sulfato de amonio presentaron una tendencia hacia una mejor respuesta a la fertilización que cuando se utilizó urca como fuente de nitrógeno.

La deficiencia de azufre en suelos tropicales de Guatemala es ampliamente conocida, de donde no es sorprendente que los tratamientos de azufre tuvieron un efecto positivo en el rendimiento. Pero, esta observación se tiene que usar con mucho cuidado, especialmente cuando se utiliza sulfato de amonio como fuente de azufre, ya que esta fuente de nitrógeno podría causar problemas en suelos con tendencia a acidez.

Las aplicaciones de ácido orgánico (ácido acético) tienen una tendencia de quebrar la estructura mineral del alófano e imogolita, lo cual permitiría reducir la fijación del fósforo en suelos de origen volcánico. Esto, aunque este efecto no fue observado en todas las localidades bajo estudio, se detectó un efecto positivo al aplicar 10 litros de ácido acético, junto con la aplicación en banda del fósforo en un suelo con un $\mathrm{pH}$ bajo.

Un concepto básico investigado fue el estimar el efecto de aplicar el sulfato de calcio junto con el fósforo en banda para precipitar el fósforo como producto reactivo DCPD y DCP (difosfato dicalcio dihidrato y fosfato dicálcico respectivamente), con lo cual se espera tener una mayor dis ponibilidad de fósforo con respecto al tiempo. La reacción inicial al tener una posible super saturación de la banda con respecto al calcio permite la formación de los productos DCPD y DCP anteriormente mencionados. Al evitar la fijación inicial del fósforo como quelato de alofano o imogolita utilizando este método de aplicación $\left(\mathrm{CaSO}_{4}+\mathrm{P}\right.$ juntos$)$ podría tener mayor impacto en áreas con deficiencias de fósforo y azufre en suelos con altos niveles de ceniza volcánica.

Como se había mencionado, las deficiencias de azufre en suelos ácidos son de mayor interés porque el uso común del sulfato de amonio podría incrementar la acidez aún más. El método propuesto de aplicación del $\mathrm{CaSO}_{4}$ (sal neutra, el cual no aumentaría la acidez del suelo) junto con el fósforo podría evitar los efectos ácidos del sulfato de amonio y al mismo tiempo aumentar la disponibilidad de] fósforo en el suelo. Investigaciones previas también han indicado que existe una interacción antagonística con respecto al azufre (iones sulfato) $\mathrm{y}$ aplicaciones de $\mathrm{P}$, especialmente en suelos donde existe una capacidad intercambiable aniónica (en este caso, $\mathrm{SO}_{4}=$ adherido al complejo intercambiable del suelo). Dado que el fósforo se fija en forma compleja con varios elementos, se puede argumentar que aplicaciones de fósforo podrían intercambiar con el $\mathrm{SO}_{4}$ en el complejo anionicio del suelo, lo cual permitiría la lixiviación del $\mathrm{SO}_{4}$.

\section{LITERATURA CITADA}

CRUZ, J. R., DE LA. 1982. Clasificación de zonas de vida de Guatemala a nivel de reconocimiento. Guatemala, Instituto Nacional Forestal. 42p.

GUATEMALA, INSTITUTO GEOGRAFICO NACIONAL. 1972. Atlas de Guatemala. Ese. varias. 45 p.

INSTITUTO DE CIENCIA Y TECNOLOGIA AGRICOLAS (Gua.). 1976. Informe Equipo Integrado de Producción "C". Guatemala. p. 46,56 ,

- - - - 1977. Informe Equipo de Prueba de Tecnología Subregión IV-3. Retalhuleu, Guatemala. p. 59, 100.

- - - 1978. Informe Técnico Equipo de Prueba de Tecnología. Parcelamiento La Máquina. Guatemala. s.p.

- - - - 1983. Informe Técnico, Equipo de Prueba de Tecnología Subregión IV-3, Retalhuleu, Guatemala. p. 68.

- - - - 1984. Informe Técnico Equipo de Prueba de Tecnología Subregión IV-3, Retalhuleu, Guatemala. p. 70.

- - - - Sondeo de los Parcelamientos Caballo Blanco, Santa Fe, El Reposo, El Rosario y Monterrey. Socioeconomía Rural. Guatemala. p. 17.

- - - 1 1985. Informe Técnico Equipo de Prueba de Tecnología Subregión IV-3, Retalhuleu, Guatemala. p. 67.

- - - 1986. Informe Técnico Equipo de Prueba de Tecnología Subregión IV-3, Retalhuleu. Guatemala. p. 47.

OLSON, R. A.; DREIER, A.F. 1956. Nitrogen, a key factor in fertilizer phosphorus efficiency. Soil Sci. Soc. Am. Proc. 20:509-514.

RAUN, W. R.; SANDER, D.H.; OLSON R.A. 1987. Phosphorus fertilizer carriers and their placement for minimum till corn under sprinkle irrigation. Soil Sci. Soc. Am. J. 51:1055-1062.

SIMMONS, C.H.; TARANO, J.M,, PMM, J.A. 1959- Clasificación de reconocimiento de suelos de la República de Guatemala. Trad. por Pedro Tirado Sulsona, Guatemala. José de Pineda Ibarra. p. 175-293.

WATANABE, Y. 1966. Nat. Inst. Agr. Sci., Japan, Bull. B 16:91-148 (J-E). 